\title{
DISCRETE ISOPERIMETRIC-TYPE INEQUALITIES ${ }^{1}$
}

\section{H. D. BLOCK}

1. Introduction. The classical isoperimetric inequality bounds the area enclosed by a curve in terms of its length. For a gridwork the analogue of area is the total number of lattice points lying inside the curve; the analogue of length is either the number of boundary lattice points, or else the sum of their multiplicities (defined in $\$ 2$ below). Inequalities relating these quantities are given in \$3. If the lattice work is constructed at different angles some of the above quantities depend on the orientation; averaging over the orientations gives average values for these. These are involved in the inequalities of $\$ 4$.

We establish inequalities bounding the area or its analogue in terms of the perimeter or its analogue. We also relate the perimeter with its analogue. Relations between the area and its analogue may be found, e.g., in $[1 ; 2 ; 3]$.

The author is indebted to Professor M. Kac and A. Calderón for many useful suggestions.

2. Definitions and notation. Let $R$ be a plane convex region with area $A$, bounded by a curve $C$ which consists of a finite number of smooth arcs the sum of whose lengths is $L$. A grid work is formed by the lines $x=n \delta, y=n \delta, n=0, \pm 1, \cdots$; the lattice points are the intersections of these lines: a lattice segment is the segment of a grid line between two successive lattice points. We assume that: $(\alpha)$ no lattice point lies on $C$; and $(\beta)$ no lattice segment intersects $C$ more than once. A lattice point in $R$ is called an interior lattice point if its four nearest neighboring lattice points all lie in $R$; otherwise it is a boundary lattice point of multiplicity $k$, where $k$ is the number of those four nearest neighbors that lie outside $R$. The sum of the multiplicities of all the boundary lattice points is denoted by $M$; the number of boundary lattice points is $N$ and the number of lattice points in $R$ is $T$. Let $W_{x}$ be the length of the horizontal projection of $C$; $W_{y}$ the length of the vertical projection. If $y=n \delta$ is the rightmost grid line to the left of $R$, and $y=m \delta$ is the leftmost grid line to the right of $R$ then we let $B_{x}=(m-n) \delta$; similarly for $B_{y}$.

3. Some inequalities. In the computations below we find inequalities involving $M$ rather than $N$; these can be converted into in-

Presented to the Society December 29, 1956; received by the editors January 16, 1957 and, in revised form, February 4, 1957.

1 Research under contract with the Office of Naval Research. 
equalities involving $N$ by noting that $(1 / 4) M \leqq N \leqq M-3$. If there is more than one lattice point in $R$ then $(1 / 3) M \leqq N \leqq M-4$. If each lattice line that contains one lattice point in $R$ contains at least one more, then $(1 / 2) M \leqq N \leqq M-4$.

As we trace around the curve $C$, each time we cross a lattice line we create one multiplicity. Since the total number of crossings is $2\left(B_{x} / \delta+B_{y} / \delta-2\right)$ we have

$$
B_{x}+B_{y}=\frac{M \delta}{2}+2 \delta
$$

(Another way to see this is to note that the number of vertical lines cutting $R$ is $B_{x} / \delta-1$ and each one yields two multiplicities; similarly for the horizontal lines).

Clearly $B_{x}-2 \delta \leqq W_{x} \leqq B_{x} ; B_{y}-2 \delta \leqq W_{y} \leqq B_{y}$ so that

$$
\text { (2) } \frac{M \delta}{2}-2 \delta=B_{x}+B_{y}-4 \delta \leqq W_{x}+W_{y} \leqq B_{x}+B_{y}=\frac{M \delta}{2}+2 \delta \text {. }
$$

Let an inscribed polygonal approximation to $C$ consist of line segments of length $\lambda_{i}$ with horizontal and vertical projections of lengths $a_{i}, b_{i}$. Then $\sum \lambda_{i} \leqq \sum\left(a_{i}+b_{i}\right)=2\left(W_{x}+W_{y}\right)$. Hence $L \leqq 2\left(W_{x}+W_{y}\right)$. On the other hand $L \geqq \sum\left(a_{i}^{2}+b_{i}^{2}\right)^{1 / 2} \geqq \sum\left(a_{i}+b_{i}\right) / 2^{1 / 2}=2^{1 / 2}\left(W_{x}+W_{y}\right)$. Hence

$$
2^{1 / 2}\left(W_{x}+W_{y}\right) \leqq L \leqq 2\left(W_{x}+W_{y}\right) .
$$

From (2) and (3) we find

$$
\frac{(M-4) \delta}{2^{1 / 2}} \leqq L \leqq(M+4) \delta .
$$

Consider the ratio $A^{1 / 2} / M \delta$. If we fix $M \delta$, then by (1) we fix $B_{x}+B_{y}$; for regions having this fixed, the area can approach at most that of a rectangle with area $B_{x} B_{y}$; this is greatest when $B_{x}=B_{y}$ $=M \delta / 4+\delta=A^{1 / 2}$. Hence

$$
M \geqq 4\left(\frac{A^{1 / 2}}{\delta}-1\right) .
$$

Similarly if we consider the ratio $T^{1 / 2} / M \delta$, then, when $M \delta$ is fixed, so is $B_{x}+B_{y}$; the greatest possible number of lattice points contained in such a region is $\left(B_{x} / \delta-1\right)\left(B_{y} / \delta-1\right)$; the maximum value of this, subject to $B_{x} / \delta+B_{y} / \delta=M / 2+2$, is $(M / 4)^{2}$. Hence

$$
M \geqq 4 T^{1 / 2}
$$


and from (4)

$$
L \geqq 8^{1 / 2} \delta\left(T^{1 / 2}-1\right) .
$$

4. Inequalities involving the averaged quantities. Let $\theta$ be an angle between 0 and $\pi$. Keeping $R$ and the $x$ and $y$ axes fixed, we form another grid work with the lines $\xi=n \delta, \eta=n \delta, n=0, \pm 1, \pm 2, \cdots$, where the $(\xi, \eta)$ axes are obtained by rotating the $(x, y)$ axes through an angle $\theta$ and then translating the origin so that assumptions $(\alpha)$ and $(\beta)$ are satisfied for the new lattice; we assume that this can be done for each $\theta$ between 0 and $\pi$. With definitions analogous to those in $\S 2$ we see that $W_{\xi}$ and $W_{\eta}$ are uniquely determined by $\theta$, while $M, B_{\xi}, B_{\eta}, N$ and $T$ are not necessarily determined uniquely. However, if for each $\theta(0 \leqq \theta<\pi)$ one selects one such grid work one obtains single valued functions $M(\theta), B_{\xi}(\theta)$, etc., as well as $W_{\xi}(\theta), W_{\eta}(\theta)$; let $\bar{M}=(1 / \pi) \int_{0}^{\pi} M(\theta) d \theta$, etc.

Now $2 W_{\xi}(\theta)=\int_{0}^{L}|\cos (\alpha(s)-\theta)| d s$, where $\alpha(s)$ is the angle between the $x$ axis and the tangent to $C$ at the point specified by the arc length parameter $s$; hence

$$
\begin{aligned}
\bar{W}_{\xi}=\bar{W}_{\eta} & =\frac{1}{\pi} \int_{0}^{\pi} W_{\xi}(\theta) d \theta=\frac{1}{2 \pi} \int_{0}^{\pi} \int_{0}^{L}|\cos (\alpha(s)-\theta)| d s d \theta \\
& =\frac{1}{2 \pi} \int_{0}^{L} \int_{0}^{\pi}|\cos (\alpha(s)-\theta)| d \theta d s=\frac{L}{\pi} .
\end{aligned}
$$

Averaging (2) we obtain

$$
\frac{\pi \bar{M} \delta}{4}-\pi \delta \leqq L \leqq \frac{\pi \bar{M} \delta}{4}+\pi \delta .
$$

Hence as $\delta \rightarrow 0, \bar{M} \delta \rightarrow 4 L / \pi$.

Note that from (5) and (8) we get $L \geqq \pi A^{1 / 2}-2 \pi \delta$; this is comparable with the classical isoperimetric inequality $L \geqq 2(\pi A)^{1 / 2}$, which is somewhat surprising considering the crude methods we have used.

Averaging (6) we get from (8)

$$
L \geqq \pi \delta\left(\overline{T^{1 / 2}}-1\right) .
$$

\section{BIBLIOGRAPHY}

1. Hilbert and Cohn-Vossen, Geometry and the imagination, New York, Chelsea, 1952, p. 33.

2. H. Steinhaus, Mathematical snapshots, New York, Stechert and Co., 1938, p. 50.

3. - Oxford University Press, 1950, p. 77.

Cornell University 